\title{
Physicians' attitudes toward race, genetics, and clinical medicine
}

\author{
Vence L. Bonham, JD ${ }^{I}$, Sherrill L. Sellers, PhD', Thomas H. Gallagher, $M D^{3}$, \\ Danielle Frank, MD, MPH $H^{4}$, Adebola O. Odunlami, $M P H^{1}$, Eboni G. Price, MD, MPH ${ }^{5}$, \\ and Lisa A. Cooper, MD, MPH
}

\begin{abstract}
Purpose: This qualitative study explored black and white general internists' attitudes about the relevance of race in clinical care; views of the relationships among race, genetics, and disease; and expectations about the future of genetics and health. Methods: We conducted 10 racially concordant focus groups of primary care physicians in five metropolitan areas in the United States. Ninety board certified or eligible general internists (50 self-identified whites and 40 self-identified blacks) participated in the study. Analysis included a two-stage independent review and adjudication process. Results: Both black and white physicians concluded that the race of the patient is medically relevant but did not agree upon why race is important in clinical decisions. They were reticent to make connections among race, genetics, and disease and asserted that genetics has a limited role in explaining racial differences in health. However, they were enthusiastic about the future of genomic medicine, believing that the main benefit will be the potential to improve the efficacy of commonly used drugs. Conclusions: Understanding the similarities and differences between black and white physicians' attitudes and beliefs about race, health and genetics is important for the translation of genomics to clinical care. Genet Med 2009:11(4): 279-286.
\end{abstract}

Key Words: clinical decision making, race, genetic variation, health disparities, physicians' attitudes

A $\mathrm{s}$ medicine enters the genomic age, fundamentally altering the course of disease in individuals may become possible. ${ }^{1}$ Genome-wide association studies and other methods to identify gene variants in common diseases and their prevalence in different population groups may hasten the application of genomic information in primary care medical practice. ${ }^{2,3}$ However, al-

From the ${ }^{1}$ Social and Behavioral Research Branch, National Human Genome Research Institute, National Institutes of Health, Bethesda, Maryland; ${ }^{2}$ School of Social Work, University of Wisconsin-Madison, Madison, Wisconsin and Department of Family Studies and Social Work, Miami University, Oxford, Ohio; ${ }^{3}$ Department of Medicine, Division of General Internal Medicine and Department of Medical History \& Ethics, University of Washington, Seattle, Washington; ${ }^{4}$ Veterans Affairs Puget Sound Health Care System, Seattle Washington; ${ }^{5}$ Section of General Internal Medicine \& Geriatrics, Tulane University Health Sciences Center, New Orleans, Louisiana; and ${ }^{6}$ Division of General Internal Medicine, Department of Medicine, Johns Hopkins University School of Medicine, Baltimore, Maryland.

Vence L. Bonham, JD, National Human Genome Research Institute, National Institutes of Health, 31 Center Drive Room B1B55, Bethesda, MD 20892. E-mail: bonhamv@mail.nih.gov.

Disclosure: The authors declare no conflict of interest.

The content is solely the responsibility of the authors and does not represent any position or policy of the National Human Genome Research Institute, National Institutes of Health or Department of Health and Human Services.

Submitted for publication August 29, 2008.

Accepted for publication November 18, 2008.

DOI: $10.1097 /$ GIM.0b013e318195aaf4 though the application of genomic information to address common diseases is still in its infancy, the use of race as a surrogate marker for describing human genetic variation and to conduct gene expression analysis is becoming common. ${ }^{4-6}$ The utility of race in clinical practice today to predict ancestry, genetics, and outcomes of treatment has been described by Barr $^{7}$ as the "practitioner's dilemma."

Some argue that racial and ethnic categories can serve as useful variables to investigate the genetic component of disease $\mathrm{e}^{8-10}$ and patients' responses to treatment. ${ }^{11,12}$ Others question the utility of race as a variable in understanding genetic variation. ${ }^{13-15}$ The debate is complex and fluid. Many social scientists and health services researchers believe that the study of race is necessary to understand the social determinants of health status and treatment; however, many are concerned that if physicians rely on race as a proxy for genetic risk and treatment decisions, this could exacerbate health care disparities and might even lead to poorer quality of care for all patients. ${ }^{16-22}$

Studies suggest that physicians consciously and subconsciously incorporate racial information about patients into their communication styles and decision making. ${ }^{23-26}$ However, no studies that we are aware of have examined clinicians' attitudes toward the relationship between race and genetics in clinical decision making.

We conducted a focus group study that addressed three questions: (1) do primary care physicians believe that race is relevant in clinical decision making? (2) what do primary care physicians believe about the relationship among race, genetics, and disease? and (3) what are primary care physicians' attitudes about the future of genomic medicine?

\section{MATERIALS AND METHODS}

\section{Study design and sample}

Between October 2005 and March 2006, we conducted 10 focus groups in five locations (Atlanta, GA; Detroit, MI; Los Angeles, CA; Philadelphia, PA; and Baltimore, MD) with general internists who self-identified their race as either black or white.

To be eligible for the study, participants had to be boardeligible or certified general internists trained in the United States who provide general and preventive health care services to patients of various racial and ethnic backgrounds. We excluded physicians who were not currently practicing medicine and were not US medical school graduates. Physicians that did not self-identify as black or white were also excluded. We focused on black and white physicians for three reasons. First, we wanted to reduce confounding effects possibly present in other possible groupings (e.g., majority/ minority). Second, as our interest was race and genetics, it was important to recruit racially diverse physicians who had a diverse patient population, and the literature suggests minority health care professionals are more likely to serve 
minority populations. ${ }^{27}$ Third, based on the theory that social context helps to shape attitudes, norms, and values, we hypothesized that black and white physicians might hold different opinions about race-related issues.

\section{Recruitment}

Recruitment occurred via personal invitation letters sent by mail and/or electronic mail to all general internists whose primary board certification was in internal medicine in the American Medical Association Physician Profile Database and to general internists on the faculty roster of the medical schools in identified counties in each focus group geographic area. Because of the limited number of black general internists in the United States, ${ }^{28}$ we also notified local chapters of the National Medical Association in each metropolitan area of the study and requested names of potentially eligible physicians. Finally, we used snowball sampling techniques as an additional strategy to recruit black physicians.

Written informed consent was obtained at the beginning of each session and a monetary incentive was provided to each physician at the end of the sessions. The study was approved by the Human Subjects Institutional Review Board of the National Human Genome Research Institute of the National Institutes of Health.

\section{Data collection}

Two general internists who are experienced qualitative researchers (T.H.G., E.G.P.) and racially concordant with the focus group participants moderated the groups. Each 2-hour focus group started with discussion of a brief clinical vignette, continued with probing of participants' perceptions of the relevance of patients' race in clinical decision making and relationships among race, genetics, and disease, and concluded with participants' completion of a brief demographic survey. (The focus group guide is available from the authors upon request.) All focus group sessions were audiotaped and transcribed verbatim for analysis.

\section{Data analysis}

Several methods were used to code and analyze the study data. A coding scheme was developed using actual words used by the participants to identify key themes in the discussions. NVivo $7 \AA$ software program was used for organizing and coding qualitative data. Two members of the research team (D.F., A.O.O.) independently coded the transcripts. Coding differences were reconciled over several meetings with the core research team. (V.L.B., D.F., A.O.O., and S.L.S.).

Several steps were employed to ensure reliability and validity of the data and analysis: (1) both moderators used consistent focus group guides for all sessions; (2) a single professional transcription agency used audiotapes and observer notes to transcribe tapes; (3) two members of the research team independently coded the data; (4) the core research team reviewed all coding of transcripts; (5) the research team resolved all inconsistencies between the two coders to create the final dataset for analyses; (6) all investigators agreed upon all themes during a series of research meetings; and (7) extra care was taken in assessing racial group differences. We explored potential differences between racial groups by comparing words, phrases, statements, and comments between groups and defined evidence of differences as the presence of one or more themes in the majority of transcripts from one racial group but not the other. When there seemed to be a racial group difference among the physicians, these themes were reviewed by the entire research team, and only those themes that, after an iterative process of analysis and reanalysis, could be agreed upon by the entire group were included in the findings.

\section{RESULTS}

As indicated in Table 1, 90 general internists participated in 10 focus groups; 5 black groups and 5 white groups. Of the total

Table 1 Characteristics of physicians

\begin{tabular}{ccc}
\hline Black & White & Total \\
$(n=40)$ & $(n=50)$ & $(n=90)$ \\
$\%$ & $\%$ & $\%$ \\
\hline
\end{tabular}

Age (yr)

$29-40$

23

22

22

$41-50$

44

32

37

$51-79$

46

40

Gender $^{a}$

Male

60

84

Female

40

Practice in University teaching 55

or residency training

environment

Years in practice (median)

Exposure to genetics in practice

Low

Medium

High

Had genetics course in

Source of genetics information $^{b}$

Professional meetings 40

CME courses

Other text resources

Other physicians

Internet

Racial/ethnic distribution of patient panel ${ }^{c}$

$\begin{array}{llll}>50 \% \text { White } & 10 & 64 & 40 \\ >50 \% \text { Black } & 73 & 20 & 43 \\ >50 \% \text { Latino/other } & 18 & 16 & 17\end{array}$

Gender of patients ${ }^{a}$

\begin{tabular}{lrrl}
$>50 \%$ Female & 73 & 54 & 62 \\
$>50 \%$ Male & 20 & 16 & 18 \\
$50 \%$ Female, $50 \%$ male & 8 & 30 & 20 \\
\hline
\end{tabular}

${ }^{a} P<0.05$

${ }^{b}$ Respondent could select more than one response. ${ }^{c} P<0.001$. 
sample, the mean age was 48 years and $73 \%$ were male. Almost half $(49 \%)$ practiced in university or residency teaching environments. The median number of years in practice was 15 . A larger majority of white physicians were male $(84 \%$ vs. $60 \%$; $P<0.05)$. A majority of white physicians reported caring for a predominantly white patient panel, whereas the majority of black physicians reported caring for a predominantly black patient panel (64\% vs. $73 \% ; P<0.001)$. A majority of physicians reported caring for female patients; the percentages were greater for black physicians ( $73 \%$ vs. $54 \% ; P<0.05)$. There were no significant differences between the two racial groups of physician participants on all remaining demographic characteristics.

The majority of the physicians $(75 \%)$ rated their exposure to genetics in their practice as "low." Nearly $70 \%$ reported having had a genetics course or curriculum of some type in medical school. Mean instruction time was estimated at 18 hours. The most common sources of genetic information were professional meetings (44\%) and continuing medical education courses (43\%).

The main themes are presented in Table 2. The table includes our research questions, main themes, and excerpts from representative quotes. The table also notes which themes differed across racial groups.

\section{Relevance of patient's race in clinical decision making}

Analysis of the first research question-is a patient's race relevant in clinical decision making-revealed three main themes: (1) physicians believed that race is a complex and poorly defined concept; (2) physicians' views on the medical

Table 2 Qualitative analysis ${ }^{a}$

\begin{tabular}{|c|c|c|c|}
\hline Research question & Salient themes & Quotes & Black/white differences \\
\hline $\begin{array}{l}\text { Is patient's race relevant } \\
\text { in clinical decision } \\
\text { making? }\end{array}$ & $\begin{array}{l}\text { Physicians believed that race } \\
\text { is a complex and poorly } \\
\text { defined concept }\end{array}$ & $\begin{array}{l}\text { "Plus there's no certain line about } \\
\text { what race is..." }\end{array}$ & $\begin{array}{l}\text { Black physicians explored the socioeconomic } \\
\text { influences on health and their relationship } \\
\text { with race for longer periods than did white } \\
\text { physicians. }\end{array}$ \\
\hline
\end{tabular}

Physicians' views of medical relevance of race varied

Physicians contended that race is one of many factors in decision making

What are the race, genetics and disease?

Physicians were reticent to make the three-way connection among race, genetics and disease relationships between

Physicians noted that most racial disparities in disease are not explained by genetics

What is expected from the future of genomic medicine?
Physicians are excited and hopeful about the promises of personalized medicine

\footnotetext{
Physicians agreed that genetics may supersede the use of race in clinical decision making

Physicians expressed concern about potential negative consequences of genomic medicine
}

"So, I don't understand how you can practice race neutral medicine in a non-race neutral world"

"There are so many different factors. To me race is just one of the many of them"

“...But we're lacking the tool, the science tool, to really focus in on the question of specific DNA variations that account for different outcomes and different diseases in various groups of people"

"I think there is a portion of it that's genetics but particularly with things like cancer, I'm going to say that I think most of it is probably environmental in access to care and treatment differences"

"I can't wait. I'm excited. You know if within ten years we can do genome testing and find the ideal medication for a given disease in a given patient that is going to revolutionize medicine."

"...And if I could genetically test my patients, doesn't that supersede knowing their race?"

"I think if you're going to use it for good, then, that's wonderful. But..."
White physicians were more likely to describe race as a culturally sensitive issue.

Black physicians referred to their own practice experiences when discussing race and socioeconomic influences to a greater extent than white physicians.

Black physicians were more likely to question whether genetic research was the most productive way to examine racial disparities in health.

No differences were found between black and white physicians.

\footnotetext{
${ }^{a}$ Representative quotes were selected through a three-part process. The core analysis team selected six to eight quotes for each theme, then the entire review team assessed the quotes, mindful of variability across speakers, and geographic region; last the core team reexamined each quote within context of the statements immediately before and after. Only quotes agreed upon by the entire research team were determined to be representative.
} 
relevance of race varied; and (3) all groups contended that a patient's race is one among many factors that influence health care decisions.

\section{Physicians believed that race is a complex and poorly defined concept}

Physicians found the concept of race to be ill defined and somewhat confusing.

Plus there's no certain line about what race is. I mean what percentage of a particular race do you have to be to be that race. Do you have a reflectometer to measure the skin color? What does it mean? (Baltimore, white)

Focus group discussions reflected the general sensitivity regarding race in the United States and the current debate about the role of race in health care. Notable issues from the discussions surrounding the complexity of race were the discomfort that physicians have while discussing race with their patients and the often vague way in which racial information is collected from their patients. There were black and white differences in physicians' levels of comfort about discussing race and race-related health issues with patients; black physicians were less likely to consider discussions of race and race-related health issues as topics they were uncomfortable with. White physicians tended to be more varied in their comfort levels. Some physicians described discomfort with these issues; others found discussions of patient's race to be straightforward.

There are people especially some of the African American patients who are always wary of the man watching over them and careful about whether we are judging them because of their skin color - accusing them of being inferior in some way. (Detroit, white)

You know if I have a question you know, that's what you're there for, and that's what they're in your office for. I ask them - what's your background? What's your ethnic background? What's your racial background? I don't think that's an oversensitive question. (Philadelphia, white)

Physicians described a variety of strategies for collecting patient's race. Many relied on visual cues coupled with knowledge of their established patient populations. Some participants questioned the value of collecting race.

. . . but I think that there are some real historical implications of race as it relates to health status of African Americans. The issue of race was used to separate African Americans and European Americans on wards. Your race identified where you would go, and what level of care you received ... One of the reasons I don't take race is because historically, I have a huge problem with how it has been used. And, I'm not sure what that marker will mean in, as I put it on the chart as it, as that chart flies through here and there. (Detroit, black)

One reason physicians may find the concept of race to be confusing and of limited clinical utility is that the relationships between race and genetics are not well specified. Participants struggled to reconcile two views: that racial categories are an objective biological division based on genetic differences and that racial categories represent solely socially defined groups.
The following quotes reflect the two dissimilar poles of view expressed in the focus groups.

So, I think we clearly know that biologically there are different chemicals and different functional processes going on in an African American versus a white patient. And I think when you're treating that patient, you have to take that into account. They're going to respond better to one drug than another perhaps. (Detroit, white)

Race is a social construct. It's useful. It's very useful, given the historic context, I would not let anybody not refer to me as a black woman. However, I actually do believe that there's no biological basis for that.. (Detroit, black)

\section{Physicians' views on why race is medically relevant varied}

Although physicians expressed the view that race is poorly defined, they nonetheless felt it was, in some way, medically relevant. Physicians offered conflicting views on the degree of relevance and the specific role of race in clinical decision making. Some physicians argued race was one of the most salient factors in health care.

So, I don't understand how you can practice race neutral medicine in a nonrace neutral world. I gotta marry those two ... (Baltimore, black)

Others argued that race was not especially relevant.

I think it may play a role. But I think it's more of a minority of cases as opposed to the majority of cases because I do think that treatment does not vary that much based on race. (Baltimore, white)

Despite varied views about the role of race, almost everyone thought race was medically relevant and useful in some way. The way physicians used race varied from gaining insights into a patient's culture to initiating discussions about performing particular diagnostic screening tests. Several physicians mentioned using race to inform decisions about screening, and the prostate-specific antigen test was mentioned most frequently. These discussions involved using race to inform decisions regarding test ordering and as a motivational communication tool to encourage patient adherence to screening recommendations.

\section{Physicians contended that patient's race is one of many factors in health care}

Physicians considered a patient's race as a "risk factor" used "equally but not preferentially" with other risk factors in making health care decisions.

You know you're going to suspect certain diseases more commonly in women than in men and you're going to treat young maybe different than old. There are so many different factors. To me race is just one of the many of them. (Baltimore, white)

In other words, a patient's race was not of the highest relevance; rather, other factors such as previous treatment, family history, physical exam, and laboratory data were more important. Physicians discussed other factors, such as lifestyle behaviors, patient beliefs, and socioeconomic factors, that are often associated with race and that they considered just as important to health. 
I think what "Jack" mentioned - trying to divide race and culture is a very hard thing because a lot of it is intertwined. A lot of the things that "Peter" is mentioning are cultural things, are access to care, socioeconomics-all those things may be more prevalent in a certain race but it's not the race itself that's the problem. (Atlanta, white)

On the question of the role of a patient's race in clinical decision making, several differences between black and white physicians were found. Specifically, white physicians more often described race as a sensitive issue.

But you still have to be careful. I mean if you tell somebody too many negatives about their 'race' or something like that, they might take it the wrong way. (Philadelphia, white)

Black physicians discussed socioeconomic influences on health and their relationship with race for longer periods and in reference to their own practice experiences to a greater extent than did white physicians.

When I think about resources and what we do know about health disparities, access to care, testing and being able to get the appropriate medication, education and support systems in place, that's something that we know, that's something that's in front of us. (Baltimore, black)

\section{Relationships among race, genetics, and disease}

Analysis of the second research question-what are the relationships between race, genetics, and disease-revealed two main themes: (1) physicians were reticent to make three-way connections among race, genetics, and disease and (2) they felt that racial disparities in health were not explained by genetics.

\section{Physicians were reticent to make connections among race, genetics, and disease}

Physicians readily made the connections between race and disease, and between race and genetics. To explain the connections, they gave several illustrations including differences in response to treatment between racial groups and different health outcomes between racial groups. However, physicians were reticent to make connections among race, genetics, and disease. Most contended that the correlation between race, genetics, and disease is not a strong one. One physician noted:

But we're lacking the tool, the science tool, to really focus in on the question of specific DNA variations that account for different outcomes and different diseases in various groups of people. (Philadelphia, white)

They articulated three main reasons for their reticence: lack of scientific tools, the increasing number of multiracial individuals, and the greater genetic variation within racial groups than between racial groups. Or as one physician stated:

So, genetics plays a huge role in all this. But my objection is that I don't think race is as useful a categorizing tool because the correlations aren't so good. There's been a lot of bleeding over between groups, through intermarriage and travel and so forth. And it's just not as exact as we would like it to be. (Atlanta, white)

\section{Physicians felt that genetics does not explain most racial disparities in disease}

Most physicians described a weak relationship between human genetic variation and race but were quick to distinguish between single gene disorders (e.g., Tay-Sachs and sickle cell disease) and common complex diseases. Although single gene disorders were used to illustrate the health differences seen between racial groups, physicians acknowledged the multifactorial determinants of most health outcomes. The role of race in common complex diseases, such as cancers, diabetes and hypertension, were also discussed but with a view that environment, rather than genetics, was most influential.

I think there is a portion of it that's genetics but particularly with things like cancer, I'm going to say that I think most of it is probably environmental in access to care and treatment differences. I don't think the majority of the difference is accounted for by genetics. I think more of it can be accounted for in that people may not have access to a physician or wait to go to a physician or when they get there it takes them longer to be diagnosed and then longer to be treated. (Atlanta, black)

The physicians asserted that genetics has a limited role in explaining racial differences in health. Thus, they believed that knowledge of genetics alone would not eliminate health disparities. Their belief was that access to treatment, quality of care received, and other environmental factors are more important health determinants, and although genetics plays a role, it is a minimal one.

On the question of the role of race, genetics, and disease, our analysis revealed that compared with white physicians, black physicians more often questioned whether genetic research was the most productive way to examine racial disparities in health and expressed concern that the appropriation of funding for public health and social and behavioral research was inadequate.

\section{Attitudes about the future of genomic medicine}

Analysis of the third research question - what are physicians' attitudes about the future of genomic medicine - revealed three main themes: (1) physicians had a general enthusiasm about the promise of personalized medicine; (2) they forecasted that genetic information will someday supersede patient's race in medical decision making; and (3) they offered a cautionary note about the potential negative consequences of genomic medicine.

\section{Physicians were enthusiastic about the promise of personalized medicine}

The majority of the physicians expressed an enthusiasm for the age of genomic medicine. One physician describes his eagerness for the future:

I can't wait. I'm excited. You know if within ten years we can do genome testing and find the ideal medication for a given disease in a given patient that is going to revolutionize medicine. And I suspect that's where we're headed. This is good stuff. (Los Angeles, white)

The majority of physicians foresaw genomics as an important part of clinical medicine in the future. They described the use of genetic information for clinical practice primarily in predictive testing. The reported examples of predictive testing fell into two main categories: tests to predict drug response and tests to identify genetic conditions such as Huntington disease and 
predisposition to breast and ovarian cancer because of mutation in BRCA 1 or 2 . The physicians believed that the main benefit will be the potential to improve the safety and efficacy of commonly used drugs.

... and I'm hoping that this whole genetic issue will bloom, bring forth additional information about certain specificity of drugs, and that maybe markers that we can identify, that I can say, that I know that this is going to be a more efficacious agent for you, be it diabetes or high blood pressure or whatever the disease process may be. (Philadelphia, black)

Although the physicians expressed excitement about the future benefits of genomic medicine, most physicians stated that genomics is not currently relevant to their practice. They acknowledged the large role genetics plays in research but did not believe genetics affected their medical practice in a meaningful way.

I think the clinical application currently is not there, and there's already trying to keep up with the clinical studies that do have application in my practice ... (L.A. white)

\section{Physicians agreed that genetics may supersede the use of race in clinical decision making}

The physicians widely agreed that, in the future, genetic knowledge will supersede the use of race in clinical decisions such as selection and dosing of medications. One physician forecasted:

I think with the identification of the human gene (sic) with the human genome project, that's going to be huge for the future, because then you will actually will be able to tailor-make medications based on a genetic profile, and make it just more personalized irregardless of what your race may or may not be. I think that is where, hopefully, we're headed in the future ... (Baltimore, black)

The majority of physicians concurred that when the ability to test patients for certain polymorphisms becomes common, the use of race as a marker in clinical decision making will largely be replaced. However, the participants contended that the reality of personalized medicine is still largely in the future.

\section{Physicians expressed concern about the potential negative consequences of genomic medicine}

The excitement around the promise of genomic medicine was tempered by concerns about the potential negative consequences of increased use of genetic information. Concerns were expressed about collection of genetic information and consequences of having it publicly available, especially for races and ethnicities that "have had very traumatic backgrounds." The physicians expressed concerns about genetic discrimination in health and life insurance. We note that the focus groups were held before the passage of the Genetic Information Nondiscrimination Act of 2008. One physician hypothesized about the consequences of having a chip containing her genetic profile:

"If you know what the "Kate" Chip says what I should use to stop smoking, great. But, what if you've got that chip that says, okay, well "Kate" is more prone to cocaine addiction, or heroine addiction, or is at the age of fifty-six going to have colon cancer, and this can all be predetermined. The cynic in me . . . (is concerned about) who controls the information, what is not, what is discoverable" (Baltimore, black).

\section{DISCUSSION}

Our study suggests that at present, physicians are faced with conflicting streams of information about the correlation of race and human genetic variation. Our findings indicate that race in the clinical setting is a confusing and poorly defined construct. Although most physicians believed patients' race had important clinical implications, no consensus emerged regarding why race was useful in the clinical encounter. This wide variation in physicians' attitudes suggests that patients may be treated differently depending on their physicians' views of what "race" means or represents. Furthermore, some physicians expressed discomfort with explicitly talking with patients about their race and how the physician was incorporating the patient's race into their clinical recommendations. Increasing physicians' ability to discuss ancestry, race, and ethnicity with patients in the examination room could help make physicians' clinical decision making more transparent to patients and enhance patient satisfaction.

Physicians were reticent to make the connection between race, genetics, and disease, because of the skepticism that a patient's race is a sufficient proxy for capturing genetic variation at the individual level. Physicians had high hopes for the future and foresaw genetics superseding race in clinical medicine. Their excitement was tempered by concerns about the potential for genetic discrimination and loss of privacy. ${ }^{29,30}$ Nonetheless, this excitement and anticipation regarding genomic medicine suggests that physicians will be a potentially receptive audience for genetics education. Interestingly, most of the physicians did not feel that genetics was particularly relevant to their current practices. Although physicians' enthusiasm about the future potential of genomic medicine suggests their interest for genetics education, targeting genetics education toward topics with high clinical relevance will be critical to engaging practicing physicians and increasing their knowledge of genetics. ${ }^{31-34}$

Although we hypothesized that differences might exist in black and white physicians' attitudes regarding race, genetics, and clinical decision making, the physicians in our study were much more similar than different. These similarities may reflect the powerful role that medical training and socialization plays in the development of health professionals' attitudes. However, during the focus groups, the black physicians discussed social determinants of health and racism with ease, whereas the white physicians expressed more discomfort with discussing race, particularly with patients in the exam room. The differences in identified themes may reflect a difference in the role race plays in the everyday lives of blacks and whites in the United States. ${ }^{35}$ Nonetheless, the shared beliefs among white and black doctors presents common ground for a much-needed discussion within the medical profession about race and its application to our developing knowledge of human genetic variation.

\section{Limitations}

Our study has several limitations. Qualitative research is ideal for exploring complex themes such as those presented in this article but cannot determine the proportion of physicians that hold any given attitude. Also, there is the potential for selection bias. We did not use a random sampling strategy and physicians who responded may have had unique interests, ex- 
periences, or networks that led to their participation in the study. Because participants were limited to a relatively small sample of black and white general internists, with an overrepresentation of academic internists, and internists from major metropolitan geographic areas the perspectives from these 10 focus groups may not be generalizable to all physicians. Another potential limitation is the possibility of response bias. Physicians may have been uncomfortable sharing their true opinions and may have responded to questions in ways that would enhance social desirability among their peers. We sought to minimize the likelihood of this type of bias in several ways: (1) by keeping the groups reasonably homogenous with regard to race and professional training; (2) by using race-concordant primary care physician moderators with no particular expertise in genetic research; (3) by using standard focus group moderation techniques to ensure participants felt comfortable sharing their opinions, and (4) by holding the groups in neutral locations.

\section{CONCLUSION}

Despite these limitations, the study makes several important contributions. The study offers a window into the perspectives of primary care physicians on the intersection of race and genetics in clinical decision making. The physicians articulated some of the ambiguity and growing controversy over the appropriateness of using racial categories in clinical practice. ${ }^{13,36}$ As with the rest of the medical/scientific community, the physicians did not come to a clear set of conclusions but did underscore the need for continued dialogue and education. They also grappled with the three-way links among race, genetics, and disease in ways reminiscent of the contemporary debates on the biological bases of race. ${ }^{18,19}$ Physicians in our study attempted to strike a balance between the emerging knowledge on race and genetics, with the knowledge of social and environmental determinates of disease. ${ }^{20,21}$ The physicians' enthusiasm for the future of genomic medicine suggests a readiness to embrace the emerging developments in genetics that are expected to have a profound impact on health and health care. ${ }^{32,34}$

The field of genetics and genomics is evolving, and we are learning new information regarding genetic variation and risk for disease. ${ }^{37}$ There is a great need for multidisciplinary research that will increase understanding of the genetic and environmental components of disease. ${ }^{38,39}$ This research will aid in the translation of genomic knowledge into clinical practice. Will a patient's race have more or less clinical utility in the genome era? As our knowledge grows, race may have less relevance in decisions that are based primarily on biochemical or physiologic processes, which may be better guided by genomic information. However, as long as social inequities and cultural differences exist, race is likely to matter in health care for a very long time.

Only with a better understanding of social and environmental determinants of health and their interaction with genetics will we be able to begin to unravel the causes of some of the racial and ethnic health disparities in the United States. ${ }^{40}$ Physicians must perform a balancing act as they obtain clinical information, including race, to guide their treatment of patients. In the future, genetic information will play a larger role in guiding these decisions. We are entering the genomic era of medicine, a time of great promise to develop new diagnostics tools and drug therapies for common diseases. The challenge will be to ensure that genomic medicine will improve the care of all patients regardless of their racial or ethnic identity.

\section{ACKNOWLEDGMENTS}

The study was supported (in part) by the Intramural Research Program of the National Human Genome Research Institute, National Institutes of Health. The authors thank Elizabeth M. Phillips and Sarah Knerr for their research assistance and Alan M. Guttmacher, MD, and Francis S. Collins, MD, PhD, (National Human Genome Research Institute) for their suggestions in preparing an early draft of this article.

\section{REFERENCES}

1. Collins FS, Green ED, Guttmacher AE, Guyer MS. A vision for the future of genomics research. Nature 2003;422:835-847.

2. Pearson TA, Manolio TA. How to interpret a genome-wide association study. JAMA 2008;299:1335-1344

3. Guttmacher AE, Collins FS. Realizing the promise of genomics in biomedical research. JAMA 2005;294:1399-1402.

4. Bamshad M. Genetic influences on health: does race matter? JAMA 2005; 294:937-946.

5. Carey LA, Perou CM, Livasy CA, et al. Race, breast cancer subtypes, and survival in the Carolina Breast Cancer Study. JAMA 2006;295:2492-2502.

6. Wallace TA, Prueitt RL, Yi M, et al. Tumor immunobiological differences in prostate cancer between African-American and European-American men. Cancer Res 2008;68:927-935.

7. Barr DA. The Practitioner's dilemma: can we use a patient's race to predict genetics, ancestry, and the expected outcomes of treatment? Ann Intern Med 2007; 143:809-815.

8. Burchard EG, Ziv E, Coyle N, et al. The importance of race and ethnic background in biomedical research and clinical practice. $N$ Engl $\mathrm{J}$ Med 2003;348:1170-1175

9. Risch N. Dissecting racial and ethnic differences. N Engl J Med 2006;354: 408-411.

10. Tang H, Quertermous T, Rodriguez B, et al. Genetic structure, self-identified race/ethnicity, and confounding in case-control association studies. Am J Hum Genet 2005;76:268-275.

11. Cohn JN. The use of race and ethnicity in medicine: lessons from the African-American Heart Failure Trial. J Law Med Ethics 2006;34:552-554.

12. Temple R, Stockbridge NL. BiDil for heart failure in black patients: the U.S. Food and Drug Administration Perspective. Ann Intern Med 2007;146:57-62.

13. Braun L, Faustro-Sterling A, Fullwiley D, et al. Racial categories in medical practice: how useful are they? PLoS Med 2007;4:1423-1428.

14. Cooper RS, Kaufman JS, Ward R. Race and genomics. $N$ Engl J Med 2003;348:1166-1170.

15. Frank R. What to make of it? The (Re)emergence of a biological conceptualization of race in health disparities research. Soc Sci Med 2007;64:1977-1983.

16. Bibbins-Domingo K, Fernandez A. BiDil for heart failure in black patients: implications of the U.S. Food and Drug Administration approval. Ann Intern Med 2007;146:52-56.

17. Braun L. Reifying human difference: the debate on genetics, race, and health. Int $J$ Health Serv 2006;36:557-573.

18. Fullwiley D. The molecularization of race: institutionalizing human difference in pharmacogenetics practice. Sci Cult 2007;161:1-30.

19. Krieger N. Stormy weather: Race, gene expression, and the science of health disparities. Am J Public Health 2005;95:2155-2160.

20. Krieger N. Refiguring 'race': epidemiology, racialized biology, and biological expressions of race relations. Int J Health Serv 2000;30:211-216.

21. Ossorio P, Duster T. Race and genetics: controversies in biomedical, behavioral, and forensic sciences. Am Psychol 2005;60:1-14.

22. Sankar P, Cho MK, Condit CM, et al. Genetic research and health disparities. JAMA 2004;291:2985-2989.

23. Cooper-Patrick L, Gallo JJ, Gonzales JJ, et al. Race, gender, and partnership in the patient-physician relationship. JAMA 1999;282:583-689.

24. Green AR, Carney DR, Pallin DJ, et al. Implicit bias among physicians and its prediction of thrombolysis decisions for black and white patients. $J$ Gen Intern Med 2007;22:1231-1238.

25. Schulman K, Berlin JA, Harless W, et al. The effect of race and sex on physicians' recommendations for cardiac catheterization. $N$ Engl J Med 1999;340:618-626.

26. van Ryn M. Research on the provider contribution to race/ethnicity disparities in medical care. Med Care 2002;40(suppl 1):I140-I151.

27. Smedley BD, Stith AY, Nelson AR, editors. Unequal treatment: confronting racial and ethnic disparities in health care. Washington, DC: National Academy Press, 2003.

28. Association of American Medical Colleges. Association of American Medical Colleges: Diversity in the physician workforce: facts \& figures 2006. Washington, DC: AAMC, 2006

29. Levy DE, Youatt EJ, Shields AE. Primary care physicians' concerns about offering a genetic test to tailor smoking cessation treatment. Genet Med 2007; 9:842-849. 
30. Hudson KL. Prohibiting genetic discrimination. N Engl J Med 2007;356: 2021-2023.

31. Trinidad SB, Fryer-Edwards K, Crest A, Kyler P, Lloyd-Puryear MA, Burke W. Educational needs in genetic medicine: primary care perspectives. Community Genet 2008;11:160-165.

32. Guttmacher A, Porteous M, McInerney J. Educating health-care professionals about genetics and genomics. Nature 2007;6:151-157.

33. Riegert-Johnson DL, Korf BR, Alford RL, et al. Outline of a medical genetics curriculum for internal medicine residency training programs. Genet Med 2004;6:543-547.

34. Suther S, Goodson P. Barriers to the provision of genetic services by primary care physicians: a systematic review of the literature. Genet Med 2003;5:70-76.

35. Nunez-Smith M, Curry LA, Bigby J, Berg D, Krumhoz HM, Bradley EH.
Impact of race on the professional lives of physicians of African descent. Ann Intern Med 2007;146:45-51.

36. Bamshad M. Meaningful policies for writing about genetics and race. Am J Med Genet A 2007;143A:971-972.

37. Feero WG, Guttmacher AE, Collins FC. The genome gets personal-almost. JAMA 2008;299:1351-1352.

38. Bonham VL, Warshauer-Baker E, Collins FS. Race and ethnicity in the genome era: the complexity of the constructs. Am Psychol 2005;60:9-15.

39. Burke W, Psaty BM. Personalized medicine in the era of genomics. JAMA 2007;298:1682-1684

40. Collins FS. What we do and don't know about 'race', 'ethnicity', genetics and health at the dawn of the genome era. Nat Genet 2004;36(suppl 11):S43-S47. 